\title{
The 9th Generation International Geomagnetic Reference Field
}

\author{
International Association of Geomagnetism and Aeronomy (IAGA), Division V, Working Group 8*
}

(Received August 16, 2003; Revised August 21, 2003; Accepted August 21, 2003)

The coefficients for the new 9th Generation International Geomagnetic Reference Field (IGRF) were finalised at the XXIII General Assembly of the International Union of Geophysics and Geodesy (IUGG), held at Sapporo in Japan in July 2003. The IGRF is a series of mathematical models of the Earth's main field and its annual rate of change (secular variation). In source-free regions at the Earth's surface and above, the main field, with sources internal to the Earth, is the negative gradient of a scalar potential $V$ which can be represented by a truncated series expansion

$$
\begin{aligned}
V(r, \theta, \lambda, t)= & R \sum_{n=1}^{n_{\max }}\left(\frac{R}{r}\right)^{n+1} \sum_{m=0}^{n} \\
& \cdot\left(g_{n}^{m}(t) \cos m \lambda+h_{n}^{m}(t) \sin m \lambda\right) P_{n}^{m}(\theta)
\end{aligned}
$$

where $r, \theta, \lambda$ are geocentric coordinates ( $r$ is the distance from the centre of the Earth, $\theta$ is the colatitude, i.e. $90^{\circ}-$ latitude, and $\lambda$ is the longitude), $R$ is the reference radius of the Earth $(6371.2 \mathrm{~km}) ; g_{n}^{m}(t)$ and $h_{n}^{m}(t)$ are the coefficients at time $t$ and $P_{n}^{m}(\theta)$ are the Schmidt semi-normalised associated Legendre functions of degree $n$ and order $m$. The coefficients are functions of time and for the IGRF they are assumed to vary at a constant rate for five-year intervals. For more details on main-field modelling the reader is referred to Chapman and Bartels (1940) and Langel (1987).

The coefficients are available from the International Association of Geomagnetism and Aeronomy (IAGA) web site www. iugg.org/IAGA and the World Data Centres listed at the end of this paper, along with software to compute magnetic field values from them. The new coefficients are the main-field coefficients for 1995.0 and 2000.0 (these are now definitive) and the secular-variation coefficients for 2000.0 2005.0. The previous (8th) generation IGRF was determined by a Task Force at the end of 1999 rather than at IUGG in July 1999 so that as many Ørsted satellite data could be incorporated as possible (Olsen et al. 2000; Lowes, 2000). Since then, much progress has been made on producing models of the Earth's magnetic field with the unprecedented amount of good quality satellite data available during the first half of the International Decade of Geopotential Research.

\footnotetext{
*Participating members: S. Macmillan, S. Maus, T. Bondar, A. Chambodut, V. Golovkov, R. Holme, B. Langlais, V. Lesur, F. Lowes, H. Lühr, W. Mai, M. Mandea, N. Olsen, M. Rother, T. Sabaka, A. Thomson, I. Wardinski.

Copy right (c) The Society of Geomagnetism and Earth, Planetary and Space Sciences (SGEPSS); The Seismological Society of Japan; The Volcanological Society of Japan; The Geodetic Society of Japan; The Japanese Society for Planetary Sciences.
}

In order to ensure that the accuracy of the IGRF reflects the high quality of available data, IAGA decided in 2001 that the 9th generation IGRF should be determined at the IUGG meeting in 2003, and that for 2000.0 the main-field coefficients should extend to degree 13 and be quoted to $0.1 \mathrm{nT}$ precision (to reflect improved instrument resolution).

As it is unusual to have a revision of the IGRF in a year not close to the epoch of a constituent main-field model and as this is the first generation of the model where a definitive set of main-field coefficients is followed by a set of secularvariation coefficients, there is a need to update the nomenclature used. Table 1 gives the new nomenclature that should be used henceforth, and gives a summary of the history of the IGRF.

It is recommended not to use the term IGRF without reference to the generation, as then it is difficult to establish which coefficients were actually used. For example, one cannot recover the original full-field data from an aeromagnetic anomaly dataset in order to tie it with adjacent surveys if one does not know which generation of the IGRF was used. It is also recommended that the full name be used, so that it is more apparent whether the output values are "predictive" and are therefore less accurate.

The term Definitive Geomagnetic Reference Field (DGRF) should not be needed by most model-users who just want geomagnetic field values for dates other than the epochs of the coefficients (i.e. most of the time!), as it leads to confusion. For example, if they use the term DGRF in 1996 (when the IGRF 7th generation (revised 1995) produces definitive values for all dates 1945.0-1990.0) it means something different than when they use it in 2004 (when the IGRF 9th generation (revised 2003) produces definitive values for all dates 1945.0-2000.0). The geomagnetic field values will be different for dates 1990.0-2000.0. In the rare case of use of individual sets of definitive coefficients, i.e. without any interpolation, from a particular generation of the IGRF, the term DGRF****, where $* * * *$ is the epoch of the coefficients, will be understood by everyone but the nomenclature in Table 1 is preferable as it covers all cases, for example "the 1995.0 coefficients from IGRF 9th generation (revised 2003)". What should be avoided amongst model-users are terms like IGRF****, again where $* * * *$ is an epoch. For example, with IGRF2000 it is not entirely clear whether this means "the 2000.0 coefficients from IGRF 8th generation (revised 1999)" or "the 2000.0 coefficients from the IGRF 9th generation (revised 2003)" or simply the "IGRF 8th generation (revised 1999)". 
Table 1. Summary of nomenclature and IGRF history.

\begin{tabular}{cccc}
\hline Full name & Short name & Valid for & Definitive for \\
\hline IGRF 9th generation (revised 2003) & IGRF-9 & $1900.0-2005.0$ & $1945.0-2000.0$ \\
IGRF 8th generation (revised 1999) & IGRF-8 & $1900.0-2005.0$ & $1945.0-1990.0$ \\
IGRF 7th generation (revised 1995) & IGRF-7 & $1900.0-2000.0$ & $1945.0-1990.0$ \\
IGRF 6th generation (revised 1991) & IGRF-6 & $1945.0-1995.0$ & $1945.0-1985.0$ \\
IGRF 5th generation (revised 1987) & IGRF-5 & $1945.0-1990.0$ & $1945.0-1980.0$ \\
IGRF 4th generation (revised 1985) & IGRF-4 & $1945.0-1990.0$ & $1965.0-1980.0$ \\
IGRF 3rd generation (revised 1981) & IGRF-3 & $1965.0-1985.0$ & $1965.0-1975.0$ \\
IGRF 2nd generation (revised 1975) & IGRF-2 & $1955.0-1980.0$ & - \\
IGRF 1st generation (revised 1969) & IGRF-1 & $1955.0-1975.0$ & - \\
\hline
\end{tabular}

The International Astronomical Union 1966 (IAU66) spheroid has been recommended for coordinate transformations when using the IGRF till now. The parameters of IAU66 are $a=6378.160 \mathrm{~km}, b=6356.775 \mathrm{~km}$. However the World Geodetic System 1984 (WGS84) datum and spheroid are more widely recognised as a standard $(a=$ $6378.137 \mathrm{~km}, b=6356.752 \mathrm{~km}$ ). Present-day satellite magnetic data are mostly positioned using WGS84 but for other data we are often unaware which datum is used. Differences in output IGRF magnetic field values at the Earth's surface are less than $1 \mathrm{nT}$ when the change of spheroid is made. It is recognised that some software already in circulation uses WGS84. It is therefore recommended that the WGS84 spheroid is used for coordinate transformations for the IGRF.

The 9th Generation IGRF coefficients were computed from candidate sets of coefficients produced by the participating members of IAGA Working Group V-8 listed in this paper. Their institutes and the many organisations involved in operating magnetic survey satellites, observatories, magnetic survey programmes and World Data Centres are to be thanked for their continuing support of the IGRF project.

\section{World Data Centres}

WDC for Solid Earth Geophysics

National Geophysical Data Center

325 Broadway

Boulder

CO 80303-3328

USA

Email: Susan.McLean@noaa.gov

http: / / www.ngdc.noaa.gov
WDC for Geomagnetism

Data Analysis Center for Geomagnetism and Space

Magnetism

Graduate School of Science, Kyoto University

Kyoto 606-8502

JAPAN

Email: iyemori@kugi.kyoto-u.ac.jp

http://swdcwww.kugi.kyoto-u.ac.jp

WDC for Geomagnetism

British Geological Survey

Murchison House

West Mains Road

Edinburgh

EH9 3LA

UK

Email:smac@bgs.ac.uk

http: / / www.geomag.bgs.ac.uk

\section{References}

Chapman, S. and J. Bartels, Geomagnetism (2 Vols), Oxford University Press, London, 1940.

Langel, R. A., Main field, in Geomagnetism Vol. 1, edited by J. A. Jacobs, Academic Press, London, 1987.

Lowes, F., The working of the IGRF 2000 Task Force, Earth Planets Space, 52(12), 1171-1174, 2000.

Olsen, N., T. Sabaka, and L. Tøffner-Clausen, Determination of the IGRF 2000 model, Earth Planets Space, 52(12), 1175-1182, 2000.

S. Macmillan (e-mail: smac@bgs.ac.uk) 